\title{
EVALUACIÓN CLÍNICA DE ÁCIDO MÁLICO/XILITOL EN AEROSOL Y ÁCIDO CÍTRICO EN AEROSOL PARA EL TRATAMIENTO DE LA HIPOSALIVACIÓN: ENSAYO CLÍNICO ALEATORIZADO
}

\section{CLINICAL EVALUATION OF A MALIC ACID/XYLITOL SPRAY AND A CITRIC ACID SPRAY FOR THE TREATMENT OF DENTINAL HYPOSALIVATION: A RANDOMIZED CLINICAL TRIAL}

\section{Bastías José ${ }^{1}$, Peragallo Sebastián², Vásquez Luciano ${ }^{3}$}

\begin{abstract}
RESUMEN
Objetivo: Evaluar la variación en el flujo salival en pacientes con xerostomía durante dos semanas del tratamiento con dos productos; un aerosol de ácido málico/xilitol y otro de ácido cítrico, en pacientes adultos.

Materiales y Métodos: Ensayo clínico controlado aleatorizado, farmacológico en fase II con triple ciego en 32 pacientes adultos. Tras la selección de pacientes con xerostomía, se ordenaron por severidad y fueron asignados en 2 grupos: (1) "aerosol de ácido málico/xilitol” y (2) "aerosol de ácido cítrico". A cada paciente se le entregó uno de los fármacos e indicaciones de uso, y fueron medidos en 3 sesiones, antes y 5 minutos después de la aplicación del fármaco utilizando el Test de Schirmer Oral.
\end{abstract}

Resultados: Para ambos grupos hubo un aumento de la Velocidad del flujo salival después de la aplicación del tratamiento. Podemos ver una diferencia del $47,3 \%$ entre la primera y la tercera sesión del grupo Ácido Málico/Xilitol, y de 28,43\% para el grupo Ácido Cítrico. Los datos se distribuyen de forma normal y que no existen diferencias estadísticamente significativas entre las medias de los deltas de velocidad de flujo entre los grupos en ninguna sesión.

Conclusión: Existe un efecto de similar magnitud generado por el uso de aerosol de ácido málico/xilitol y el ácido cítrico en la secreción de saliva en pacientes adultos con hiposalivación.
1. Centro de Salud Familiar Dr. Enrique Dintrans Ávila, Rancagua, Chile.

2. Servicio de Atención Primaria de Urgencia Quebrada Verde, Valparaíso, Chile.

3. Atención Privada Odontológica, San Antonio, Chile.

Correspondencia:

Bastías Valenzuela, José Tomas

Correo electrónico:

jtbastias06@gmail.com

\section{PALABRAS CLAVES:}

Ácido Málico/Xilitol; Ácido Cítrico;

Hiposalivación; Tratamiento

KEYWORDS:

Xylitol/Malic acid; Citric acid; Hyposalivation; Treatment 


\begin{abstract}
Objective: To evaluate difference in salivary flow in adult patients with xerostomia during a two weeks treatment using two products; a spray of malic acid/xylitol and another of citric acid.
\end{abstract}

Materials and Methods: Randomized Controlled Clinical Trial, phase II pharmacological evaluation with triple blind in 32 adults. After the selection of patients with xerostomia, they were ordered by severity and assigned into 2 groups: (1) "spray of malic acid/xylitol" and (2) "a spray of citric acid". Each one was given one of the drugs and use instructions, measurements were registered in 3 sessions: Prior and 5 minutes after the application of the drug using the Oral Schirmer Test.

Result: Both groups registered an increase in salivary flow after the treatment therapy. A difference of $47,3 \%$ on the salivary flow was observed among first and third week of treatment on the malic acid/xylitol spray group, meanwhile a $28,43 \%$ was observed on the citric acid spray group.

All data obtained was distributed in a normal way so that there was not any statistical significant difference among flow rate delta stockings on the groups in any session.

Conclusion: There is a similar effect on the salivary flow generated by the use of acid/xylitol spray and the citric acid spray on adult patients with hyposalivation.

\section{INTRODUCCIÓN}

La hiposalivación o hiposialia es un problema en la salud oral, que se define como disminución o ausencia de la secreción salival. ${ }^{1}$ En las personas que lo presentan, puede producir incomodidad al hablar y a la deglución, dolor e irritación de las mucosas, dificultar el uso de prótesis removible, causar halitosis, entre otros. $^{2}$

$\mathrm{Su}$ etiología es multifactorial, entre las principales causas se puede encontrar el uso de medicamentos, terapias oncológicas, terapia quirúrgica, alteraciones psicológicas y enfermedades autoinmunes. ${ }^{3}$ El $50 \%$ de los pacientes que lo padecen no presentan síntomas, ya que se puede llegar a perder hasta un $50 \%$ del flujo salival sin ser percibible. ${ }^{4}$

Según Guggenheimer et al., la prevalencia de esta enfermedad es variable rondando de un 20 a $30 \%{ }^{5}$, alcanzando casi un $100 \%$ en pacientes con Síndrome de Sjögren y aquellos en radioterapia de cabeza y cuello. ${ }^{6}$

Los pacientes que padecen de esta patología generalmente consultan por una sensación de boca seca, síntoma llamado xerostomía ${ }^{7}$; el signo que evidencia esto es la disminución cuantitativa de la saliva, es decir, la hiposalivación.

La saliva artificial del recetario magistral ha sido la alternativa terapéutica más indicada para el tratamiento de la xerostomía, debido a la simplicidad de elaboración, bajo costo y efectividad. El aerosol de ácido málico/xilitol tiene una consistencia más acuosa, diferente compuesto activo y una forma de aplicación más cómoda, ofreciendo así una innovadora alternativa terapéutica.

La relevancia del manejo de la hiposalivación radica en el impacto que puede tener en la salud oral de los pacientes, donde se puede observar un aumento del riesgo cariogénico, enfermedad periodontal, candidiasis, entre otras patologías. Sumado a que a pesar de que existe una amplia gama de tratamientos, no hay evidencia científica que muestre diferencias entre éstas, tanto en el manejo de los signos y síntomas clínicos, como del costo.

Por lo tanto, se busca evaluar la influencia del uso de estos tratamientos en la variación del flujo salival para saber finalmente si existe una diferencia significativa del efecto entre estos dos tratamientos. Se busca evaluar el flujo salival a las dos semanas de tratamiento con dos productos por separado: aerosol de ácido málico/xilitol y saliva artificial de ácido cítrico, en pacientes adultos que presentan xerostomía atendidos en la Escuela de Odontología de la Universidad de Valparaíso durante el año 2016. 


\section{MATERIALES Y MÉTODO}

Estudio de tipo analítico experimental farmacológico fase II, donde se utilizó dos fármacos en grupos de pacientes que padecen la enfermedad. El diseño utilizado correspondió a un ensayo clínico controlado aleatorizado en paralelo de equivalencia con triple ciego.

Se aplicó el triple ciego de la siguiente forma: durante el estudio, el clínico no tuvo acceso a la ficha clínica, los envases que contenían cada tratamiento no permitían saber al paciente cuál utilizaba, y los datos sin identificación no permitieron al estadístico conocer a qué paciente ni a qué grupo pertenecían.

La población correspondió a todas aquellas personas que hayan sido atendidas en la Facultad de Odontología de la Universidad de Valparaíso durante los años 2014 y 2015.

La selección de los pacientes se realizó como un muestreo no probabilístico por agrupación decisional, con una asignación aleatoria simple a los grupos. Luego de aplicado los resultados, se calculó la potencia estadística para la prueba Anova de medidas repetidas, obteniendo un 0,9 , valor alto según Cohen. ${ }^{8}$

En cuanto a los criterios de selección se consideraron pacientes entre 40 y 70 años, que respondieron positivamente la primera pregunta de la encuesta de "Fox, Busch y Baum 1987" para Xerostomía: "Siente la boca seca usualmente". 9

Fueron excluidos todos aquellos pacientes que padecieran alguna enfermedad que no permitiera un correcto examen clínico, con enfermedades sistémicas no controladas o Síndrome de Sjögren; pacientes bajo tratamiento para xerostomía previo, con dificultades motoras o cognitivas severas o con enfermedad mental degenerativa avanzada.

$\mathrm{Si}$ durante el estudio algún paciente abandonase el tratamiento o se excluye por los criterios previamente expuestos, se le reemplazaría por un nuevo paciente dentro de la fecha especificada como límite y se contactaría al paciente que seguía en severidad. Las variables utilizadas en esta investigación fueron: edad, rango etario de "40 a 49", "50 a 59" y “60 a 70" años, sexo, flujo salival definido como la cantidad de saliva por unidad de tiempo (velocidad) secretada por las glándulas salivales mayores y menores, medida utilizando "Test de Schirmer Oral". ${ }^{10}$ Uso de fármacos que causan xerostomía (sí o no),cantidad de fármacos agrupándolos en rangos de "1 a 2", "3 a 4", "5 a 6" y "7 o más", consumo de alcohol, cantidad de consumo de alcohol en rangos "1 vez al mes", "4 veces al mes" y "más de 4 veces al mes", cantidad de enfermedades sistémicas agrupándose de "1 a 2", "3 a 4" y "5 o más", presencia de alergias, uso de tabaco, cantidad de tabaco de "1 a 5", "6 a 10", "11 a 15" y "16 a 30" cigarros diarios.

Los pacientes fueron contactados, se les explicó el estudio, se preguntó si les interesaba participar y se les realizó la primera pregunta de la "Encuesta de Xerostomía. Fox, Busch y Baum 1987", correspondiente a "Siente la boca seca usualmente".

Los pacientes fueron citados para realizar un examen clínico completo y contestar el resto de la encuesta. Las respuestas se compararon y se ordenaron los pacientes según severidad.

Luego de ser aplicados los criterios de inclusión y exclusión, se obtuvo un total de 30 pacientes, los que se dividieron de forma aleatoria, en 2 grupos; un primer grupo, que utilizó un aerosol de ácido málico/xilitol (Xeros Spray®) y el otro utilizó un aerosol de ácido cítrico. Ambos usaron el producto por 14 días, junto con indicaciones de uso y dieta y se realizaron controles clínicos los días 0, 7 y 14 .

La saliva artificial de recetario magistral utilizada fue formulada con $20 \mathrm{ml}$ de solución de Metilcelulosa al 4\%, $10 \mathrm{ml}$ de glicerina, 1 gota de aceite de limón, y suero salino suficiente para completar $90 \mathrm{ml}$ de solución.

Por otro lado, el Xeros Spray® contiene ácido 
málico $1,00 \%$ el cual estimula la producción natural de saliva sin dañar el esmalte, xilitol $10,00 \%$ que hidrata la cavidad bucal, actúa contra las caries y remineraliza el esmalte, y finalmente fluoruro sódico $0,05 \%$, cuya función es prevenir la aparición de caries y remineralizar el esmalte(225 ppm). ${ }^{11}$

El test utilizado para medir el flujo salival fue el Test de Schirmer Oral. ${ }^{10}$ Como instrumento se utilizó una tira de papel absorbente milimetrada que presenta una tinción azul. Éste se aplicó en 2 oportunidades en cada sesión: la primera antes y la segunda 5 minutos después de usar 4 pulverizaciones del fármaco.

Para el uso del test se capacitó al clínico sobre la estandarización del test.

Se registraron todos los datos personales y la cuantificación del flujo salival en una ficha.

Se construyó una base de datos con los resultados en Microsoft Excel y se aplicó un análisis estadístico en el software "STATA 15.0". Las pruebas estadísticas utilizadas fueron: T de Student y ANOVA de medidas repetidas con error intrasujeto; en ambas existe un nivel de confianza del $95 \%$ y una potencia estadística del $85 \%$.

Para obtener conformidad en cuanto a los aspectos éticos nos basamos en la aplicación de los principios de beneficencia, autonomía, justicia y no maleficencia de la Declaración de Helsinki del año 2008. ${ }^{12}$

Al paciente se le explicó de forma acuciosa el consentimiento informado, se les explicó que la participación en el estudio es voluntaria pudiendo abandonar el estudio cuando lo desee, además de la protección a la intimidad y confidencialidad de los datos, utilizados únicamente para fines de investigación docencia y sin fines de lucro.

Se detallaron todos los riesgos y beneficios, tanto individuales como colectivos, resultantes de la participación. Todos los pacientes que fueron partícipes del estudio recibieron tratamiento para la xerostomía ya sea con saliva artificial, producto normalmente recomendado por la Facultad de Odontología Universidad de Valparaíso o con el producto Xeros Spray®, un producto especialmente desarrollado por laboratorios Dentaid®.

\section{RESULTADOS}

La muestra total de este estudio fue de 30 personas seleccionadas y divididas aleatoriamente en dos grupos para realizar el ensayo, donde el primer grupo ocupó Ácido Málico/Xilitol y el segundo grupo ácido Cítrico.

En el estudio, 28 personas resultaron ser del sexo femenino $(93.3 \%)$ y 2 personas correspondían al sexo masculino $(6.7 \%)$.

En lo que respecta a las enfermedades sistémicas presentes en un $86,7 \%$ de la muestra total. Un $69,2 \%$ tienen de 1 a 2 enfermedades, $30,8 \%$ presentan de 3 a 4 enfermedades y $7,7 \%$ tienen 5 o más y $26,7 \%$ de los participantes presenta algún tipo de alergia.

De los 30 pacientes, $76,7 \%$ consumen fármacos. Donde 47,8\% consumen de 1 a 2 fármacos, 34,8\% consumen de 3 a 4, 8,7\% consumen de 5 a 6 y $8,7 \%$ consumen de 7 o más fármacos.

Para ambos grupos hubo un aumento de la Velocidad del flujo salival después de la aplicación del tratamiento. Podemos ver una diferencia del $47,3 \%$ entre la primera y la tercera sesión del grupo Ácido Málico/Xilitol, y de $28,43 \%$ para el grupo Ácido Cítrico.

En análisis estadístico de medias del flujo salival se buscó encontrar las diferencias estadísticamente significativas entre grupos para las medias de los Deltas de velocidad de flujo salival antes y 5 minutos después de aplicado el tratamiento en cada sesión mediante la prueba de T-Student.

Para realizar la prueba paramétrica, se cumple con los supuestos de distribución normal mediante la prueba de "Shapiro-Wilk" y de homocedasticidad mediante la prueba de 
"Robvar", donde los p-Valores para ambas pruebas fueron mayor 0.0005 que permiten cumplir estos supuestos.

Como todos los datos son mayores a 0,05 se puede concluir que los datos se distribuyen de forma normal y que no existen diferencias estadísticamente significativas entre las medias de los deltas de velocidad de flujo entre los grupos en ninguna sesión. Por lo tanto, respecto al aumento en el flujo salival, ácido Málico/Xilitol y ácido Cítrico tienen el mismo efecto.

En cuanto al análisis estadístico de varianza de flujo salival, se desea buscar la razón determinante del aumento crónico en la velocidad de flujo salival previamente demostrada en las tablas anteriores para ambos grupos (Tabla I y II). Para esto se somete los datos a una prueba de significancia estadística "Anova de Medidas Repetidas con Error Intrasujeto" para encontrar el o los factores que afectan al comportamiento.

Los factores a analizar son el tiempo determinado con la variable "Sesión" y la variable "Paciente" como unidad que podría estar determinando la mejoría de los resultados de forma acumulativa. Se asume que las variables son dependientes.

Como todos los datos son mayores a 0,05 se puede concluir que los datos se distribuyen de forma normal.

En cuanto al ANOVA, si el P-valor es menor a 0,05 , nos dice que ese factor afecta al comportamiento en la evolución del flujo salival a través de las sesiones.

Analizando la tabla, el factor grupo arroja un mayor valor a 0,05 , mientras que el factor "tiempo" nos arroja un valor menor correspondiente a 0,0069. Por lo tanto, el tiempo sí es un factor influyente en el comportamiento evolutivo del flujo salival a diferencia del factor paciente.
Tabla I: Comparación de velocidad de flujo salival antes y después de la aplicación durante el estudio y su diferencia en las Medias en el Grupo Ácido Málico/Xilitol

\begin{tabular}{|c|c|c|}
\hline \multicolumn{3}{|c|}{ Velocidad de Aplicación } \\
\hline Sesión & $\begin{array}{c}\text { Pre-Aplicación } \\
\overline{\mathrm{x}} \pm \mathrm{mm} / \mathrm{min}\end{array}$ & $\begin{array}{c}\text { Post-Aplicación } \\
\overline{\mathrm{x}} \pm \mathrm{mm} / \mathrm{min}\end{array}$ \\
\hline Sesión 1 & $4.67 \pm 3.18$ & $6.24 \pm 3.57$ \\
\hline Sesión 2 & $6.07 \pm 4.11$ & $6.87 \pm 3.35$ \\
\hline Sesión 3 & $6.89 \pm 3.77$ & $6.82 \pm 3.04$ \\
\hline $\begin{array}{c}\text { Diferencias de Medias de velocidad Pre y Post } \\
\text { Aplicación }\end{array}$ \\
\hline \multicolumn{2}{|c|}{ Sesión } & $\%$ \\
\hline \multicolumn{2}{|c|}{ Sesión 1} & +33.80 \\
\hline \multicolumn{2}{|c|}{ Sesión 2} & +13.18 \\
\hline Sesión 3 & +13.54 \\
\hline \multicolumn{2}{|c|}{}
\end{tabular}

Tabla II: Comparación de velocidad de flujo salival antes y después de la aplicación durante el estudio y su diferencia en las Medias en el Grupo Ácido Cítrico

\begin{tabular}{|c|c|c|}
\hline \multicolumn{3}{|c|}{ Velocidad de Aplicación } \\
\hline Sesión & $\begin{array}{c}\text { Pre-Aplicación } \\
\overline{\mathrm{x}} \pm \mathrm{mm} / \mathrm{min}\end{array}$ & $\begin{array}{c}\text { Post-Aplicación } \\
\overline{\mathrm{x}} \pm \mathrm{mm} / \mathrm{min}\end{array}$ \\
\hline Sesión 1 & $5.24 \pm 3.57$ & $5.91 \pm 3.57$ \\
\hline Sesión 2 & $6.56 \pm 4.14$ & $6.98 \pm 3.80$ \\
\hline Sesión 3 & $6.73 \pm 4.05$ & $6.91 \pm 3.16$ \\
\hline
\end{tabular}

Diferencias de medias de velocidad Pre y Post Aplicación

\begin{tabular}{|c|c|}
\hline Sesión & $\%$ \\
\hline Sesión 1 & +12.71 \\
\hline Sesión 2 & +6.44 \\
\hline Sesión 3 & +2.64 \\
\hline
\end{tabular}

\section{DISCUSIÓN}

La muestra fue filtrada a través de criterios de selección, que incluían resultados positivos en la encuesta de Fox, que tiene una amplia utilización a lo largo de la historia en lo que respecta el síndrome de boca seca. ${ }^{13}$ Luego de seleccionados los sujetos de estudio, estos fueron repartidos de forma aleatoria $y$ equitativa, obteniendo 2 grupos de igual tamaño.

Para medir el flujo salival se utilizó el test de Schimer Oral, el cual fue aplicado por un solo examinador, debidamente calibrado con un Gold Standard y está validado en Estados Unidos por Chen para medir Flujo Salival. ${ }^{14}$ 
Tabla III: Promedio, desviación estándar, $t$-student de los deltas de velocidad 1, 2 y 3 correspondientes a la primera, segunda y última sesión respectivamente.

\begin{tabular}{|l|l|r|r|r|}
\hline Grupo & \multicolumn{1}{|c|}{$\mathbf{\Delta 1}$} & \multicolumn{1}{|c|}{$\mathbf{2}$} & \multicolumn{1}{c|}{$\mathbf{3}$} \\
\hline \multirow{2}{*}{$\begin{array}{l}\text { Ácido } \\
\text { Málico/Xilitol }\end{array}$} & Promedio & $-1,578$ & $-0,8$ & $-0,933$ \\
\cline { 2 - 5 } Ácido Cítrico & Desviación Estándar & 1,640 & 2,099 & 3,035 \\
& Promedio & $-0,667$ & $-0,422$ & $-0,178$ \\
\cline { 2 - 5 } & Desviación Estándar & 2,516 & 2,550 & 2,472 \\
\hline \multirow{2}{*}{ Total } & Promedio & $-1,122$ & $-0,611$ & $-0,556$ \\
\cline { 2 - 5 } & Desviación Estándar & 2,138 & 2,306 & 2,746 \\
\hline T-Student & p-valor & 0.2500 & 0.6616 & 0.461 \\
\hline
\end{tabular}

Tabla IV: Promedio, desviación estándar, ANOVA de las velocidades iniciales de las sesiones 1, 2 y 3, correspondientes a la primera, segunda y última sesión respectivamente. R2 Bondad de Ajuste.

\begin{tabular}{|c|c|c|c|c|}
\hline \multicolumn{2}{|l|}{ Grupo } & Sesión 1 & Sesión 2 & Sesión 3 \\
\hline \multirow{2}{*}{$\begin{array}{l}\text { Ácido } \\
\text { Málico/Xilitol }\end{array}$} & Promedio & 4,667 & 6,067 & 6,889 \\
\hline & Desviación Estándar & 3,187 & 4,119 & 3,771 \\
\hline \multirow[t]{2}{*}{ Ácido Cítrico } & Promedio & 5,244 & 6,556 & 6,733 \\
\hline & Desviación Estándar & 3,578 & 4,142 & 4,059 \\
\hline \multirow[t]{2}{*}{ Total } & Promedio & 4,956 & 6,311 & 6,811 \\
\hline & Desviación Estándar & 3,342 & 4,066 & 3,850 \\
\hline \multicolumn{4}{|l|}{ Modelo p-valor } & 0,000 \\
\hline \multicolumn{4}{|l|}{ Paciente } & 0.8059 \\
\hline \multicolumn{4}{|l|}{ Sesión } & 0.0069 \\
\hline \multicolumn{4}{|c|}{ Interacción Grupo/Pacientes } & 0.7901 \\
\hline \multicolumn{4}{|l|}{$\mathrm{R}^{2}$} & 0.7794 \\
\hline
\end{tabular}

Como hallazgo principal del estudio, se obtuvo que en ambos grupos se produjo un aumento del flujo salival después del tratamiento, sin embargo como estos no son estadísticamente significativos, podemos afirmar que el ácido málico/xilitol y el ácido cítrico, tienen el mismo efecto en el aumento del flujo salival, tanto en el tratamiento inmediato como mediato. No se encontraron estudios similares para ser comparado con los resultados, pero se explicaría porque ambos compuestos activos tendrían un efecto de similar magnitud en la secreción de saliva. ${ }^{15}$

Al utilizar ambos aerosoles se observó un incremento significativo en el flujo salival pero sin diferencias significativas entre ellos. En un estudio de Gómez-Moreno et al. en pacientes que tomaban antidepresivos, concluye que hubo incremento en la cantidad de saliva secretada en pacientes que utilizaron el aerosol de ácido málico/xilitol. ${ }^{16}$ AguilarSalvatierra et al, estudió pacientes con xerostomía inducida por fármacos, llegando a las mismas conclusiones. ${ }^{17,18}$

Esto podría estar explicado por el hecho de que al tener más frecuentemente un líquido estimulante en boca hace que los receptores que se ubican en la cavidad oral se activen y estimulen el sistema nervioso parasimpático (sistema que regula la secreción de fluido), aumentando así la producción de saliva crónicamente.

Dentro de las limitaciones del estudio, se realizó el examen clínico en distintos horarios, dependiendo de la disponibilidad de los pacientes y del clínico. Se sugiere realizar el examen clínico siempre a la misma hora y en horario AM para estandarizar la influencia del momento del día en la estimulación basal de 
saliva en futuros estudios.

La muestra fue seleccionada por conveniencia, sin embargo, la buena potencia estadística obtenida nos comprueba la suficiencia de sujetos considerados en el estudio. Sin embargo, se sugiere realizar cálculo de tamaño muestral para que tuviese la suficiente validez para extrapolar el resultado a otras poblaciones.

Buscando el mejor tratamiento para el paciente, se sugiere comparar estos productos con otros tratamientos, como por ejemplo la saliva artificial de preparación casera, compuesta de: $30 \mathrm{~g}$ de semilla de linaza y 1 mg de manzanilla para un litro de agua. ${ }^{19}$

Al no observarse diferencias estadísticamente significativas, el factor monetario adquiere mayor relevancia clínica, siendo la saliva artificial una alternativa más económica.

\section{CONCLUSIONES}

Respecto a la medición del flujo salival con Test de Schirmer Oral en el grupo que usó aerosol de ácido málico/xilitol y el del aerosol de ácido cítrico, se observa un aumento mediato e inmediato. Por lo que no se observan diferencias significativas en el aumento de la saliva medida con test de Schirmer oral entre ambos grupos.

Esto se debe a que el tipo de sialogogo no es tan relevante al momento de generar la saliva. La importancia radica más en tener al menos uno de los dos y no uno por sobre el otro.

Ambos productos tienen el mismo impacto en la hiposalivación ya que aumentan el flujo salival sin diferencias estadísticamente significativas entre ambos. Al presentar no presentar diferencias estadísticamente significativas con el tratamiento comúnmente utilizado (aerosol de ácido cítrico), nos permite presentar el aerosol de ácido málico/xilitol como una nueva alternativa de tratamiento para pacientes con hiposalivación.

\section{CONFLICTOS DE INTERÉS}

Sin conflictos de interés.

\section{FINANCIAMIENTO}

Autofinanciado.

\section{REFERENCIAS}

[1] Silvestre Donat F, Miralles Jordá L, Martínez Mihi V. Tratamiento de la boca seca: puesta al día [Internet]. Scielo.isciii.es. 2004. Available from:

http://scielo.isciii.es/scielo.php?script=sci_artte xt\&pid=S1698-44472004000400001.

[2] Fox P. Saliva composition and its importance in dental health, Compend Contin Educ Dent. 1989; 13:457-60.

[3] Blanco Carrión A, López López J, Peñamaría Mallón M, Seoane Lestón JM. Curso de xerostomía. Clínica de la Salud Campus Medynet.

[4] Rodríguez Palacios J, Martínez Naranjo T. La Xerostomía en pacientes con prótesis dental [Internet]. Scielo.sld.cu. 2008. Available from: http://scielo.sld.cu/scielo.php?script=sci_arttex $\mathrm{t} \& \mathrm{pid}=\mathrm{S} 0034-75072008000200010$

[5] Guggenheimer J, Moore P. Xerostomia: etiology, recognition and treatment.J Am Dent Assoc.2003 Jan; 134(1):61-9; quiz 118-9.

[6] Mravak-Stipetić M. Xerostomia-Diagnosis and treatement. Zagreb, Croacia: University of Zagreb. 2012.

[7] González Jiménez E, Aguilar Cordero MJ, Guisado Barrilao R, Tristán Fernández JM, García López PA et al. Xerostomía: Diagnóstico y Manejo Clínico. España: 2008.

[8] Cohen J. Statistical Power Analysis for the Behavioral Sciences. Hoboken: Taylor and Francis; 2013.

[9] Fox PC e. Subjective reports of xerostomia and objective measures of salivary gland performance. - PubMed - NCBI [Internet]. Ncbi.nlm.nih.gov. 1987. Available from: https://www.ncbi.nlm.nih.gov/pubmed/347759 5 DOI: $10.1016 / \mathrm{s} 0002-8177(87) 54012-0$

[10] López-Jornet P e. A simple test for salivary gland hypofunction using Oral Schirmer's test. - PubMed - NCBI [Internet]. Ncbi.nlm.nih.gov. $2006 . \quad$ Available from: https://www.ncbi.nlm.nih.gov/pubmed/165197 73

[11] Xeros Dentaid Spray [Internet]. Dentaid.cl. 2019. Available from: https://www.dentaid.cl/xeros-dentaid/xerosdentaid-spray/id27 
[12] Iacs.aragon.es. Declaracion de Helsinki de la AMM - Principios éticos para las investigaciones médicas en seres humanos. [Disponible enhttp://www.iacs.aragon.es/econocimiento/d ocumentos/ceica/2013-declaracion-helsinkibrasil.pdf;actualizado en marzo 2014]

[13] Thomson WM e. The Xerostomia Inventory: a multi-item approach to measuring dry mouth. - PubMed - NCBI [Internet]. Ncbi.nlm.nih.gov. 1999 [cited 8 August 2019]. Available from: https://www.ncbi.nlm.nih.gov/pubmed/10697 349

[14] Austin Chen, Yolanda Wai, Linda Lee, Stephen Lake, Sook-Bin Woo. Using the modified Schirmer test to measure mouth dryness

[15] Dentaid L. Laboratorios Dentaid Xerostomia: Laboratorios Dentaid; [Available from:http://www.dentaid.cl/xerostomia.]

[16] Dentaid Profesional - ESTUDIO [Internet]. Dentaid.cl. 2011]. Available from: https://www.dentaid.cl/pro/estudiosclinicos/eficacia-del-spray-de-acido-malico-1xeros-dentaid-spray-en-el-tratamiento-de-laxerostomia-inducida-por-farmacos/id3

[17] Gómez-Moreno G e. Evaluation of the efficacy of a topical sialogogue spray containing malic acid $1 \%$ in elderly people with xerostomia: a double-blind, randomized cli... - PubMed - NCBI [Internet]. Ncbi.nlm.nih.gov. 2013 Available from: https://www.ncbi.nlm.nih.gov/pubmed/23294 350. DOI: $10.1111 /$ ger.12034

[18] Gómez-Moreno G1, Aguilar-Salvatierra A, Guardia J, Uribe-Marioni A, Cabrera-Ayala $\mathrm{M}$, et al. The efficacy of a topical sialogogue spray containing $1 \%$ malic acid in patients with antidepressant-induced dry mouth: a double-blind, randomized clinical trial. Depress Anxiety. 2013 Feb;30(2):137-42. Epub 2012 Nov 1. doi: 10.1002/da.22017

[19] Irene Morales Bozo, Ana Ortega Pinto, Gonzalo Rojas Alcayaga, Juan Pablo Aitken Saavedra, Juana Olga Salinas Flores et al. Reporte preliminar sobre el efecto de un sustituto salival a base de manzanilla (Matricaria chamomilla) y linaza (Linum (usitatissimum) en el alivio de la xerostomía en adultos mayores. Trabajo de investigación. Rev Clin Periodoncia Implantol Rehabil Oral. 2015;8(2):144---149.

\section{CÓMO CITAR ESTE ARTÍCULO}

Bastías J, Peragallo S, Vásquez L. Evaluación clínica de ácido málico/xilitol en aerosol y ácido cítrico en aerosol para el tratamiento de la hiposalivación: ensayo clínico aleatorizado. Appli. Sci. Dent. 2020; 1(1): 26-33.

DOI: $10.22370 /$ asd.2020.1.1.2110 